\title{
Characterizing a Wake-Free Safe Zone for the Simplified Aircraft-based Paired Approach Concept
}

\author{
Nelson M. Guerreiro ${ }^{1}$ \\ ATK Space Systems, Inc., Hampton, VA, 23681-2199 \\ Kurt W. Neitzke², Sally C. Johnson ${ }^{3}$, H. Paul Stough, III $^{4}$, Burnell T. McKissick ${ }^{5}$ \\ NASA Langley Research Center, Hampton, VA, 23681-2199 \\ and \\ Hazari I. Syed ${ }^{6}$ \\ Raytheon Information Solutions, Hampton, VA, 23681-2199
}

\begin{abstract}
The Federal Aviation Administration (FAA) has proposed a concept of operations geared towards achieving increased arrival throughput at U.S. Airports, known as the Simplified Aircraft-based Paired Approach (SAPA) concept. In this study, a preliminary characterization of a wake-free safe zone (WFSZ) for the SAPA concept has been performed. The experiment employed Monte-Carlo simulations of varying approach profiles by aircraft pairs to closelyspaced parallel runways. Three different runway lateral spacings were investigated $(750 \mathrm{ft}, 1000$ $\mathrm{ft}$ and $1400 \mathrm{ft}$ ), along with no stagger and $1500 \mathrm{ft}$ stagger between runway thresholds. The paired aircraft were flown in a leader/trailer configuration with potential wake encounters detected using a wake detection surface translating with the trailing aircraft. The WFSZ is characterized in terms of the smallest observed initial in-trail distance leading to a wake encounter anywhere along the approach path of the aircraft. The results suggest that the WFSZ can be characterized in terms of two primary altitude regions, in ground-effect (IGE) and out of ground-effect (OGE), with the IGE region being the limiting case with a significantly smaller WFSZ. Runway stagger was observed to only modestly reduce the WFSZ size, predominantly in the OGE region.
\end{abstract}

\section{Introduction}

$\mathrm{T}$ HE nation's airspace is becoming increasingly crowded as demand for passenger air travel and cargo flights increases. As a result of this increased demand, and because of the correlated increase in the number of commercial flights, many of the nation's airports are forecast to see rising arrival and departure delays in the near future ${ }^{1,2}$. A considerable part of these increased airport delays is due to the available throughput of the airport runways.

The Federal Aviation Administration (FAA) has proposed a concept of operations geared towards achieving increased arrival throughput at U.S. Airports ${ }^{3,4}$, which either currently have closely-spaced parallel runways, or have room to add them later. Known as the Simplified Aircraft-based Paired Approach (SAPA) concept, it is a procedure-based concept that allows dependent parallel instrument approaches, under Instrument Meteorological Conditions (IMC), to airports with closely-spaced parallel runways with centerline spacing between 750 and 2500 feet. A primary safety concern with the SAPA concept is the risk that one of the aircraft will experience a dangerous encounter with the wake vortices generated by the other, along the paired approach. Under current rules, independent approaches are allowed to parallel runways with centerline spacing of 4300 feet or greater (or down to

\footnotetext{
${ }^{1}$ Research Engineer, Aeronautics Systems Analysis Branch, Mail Stop 442, member AIAA.

${ }^{2}$ Senior Analyst, Aeronautics Systems Analysis Branch, Mail Stop 442, member AIAA.

${ }^{3}$ Senior Research Engineer, Crew Systems \& Aviation Operations Branch, Mail Stop 152.

${ }^{4}$ Senior Research Engineer, Crew Systems \& Aviation Operations Branch, Mail Stop 156A, senior member AIAA.

${ }^{5}$ Mathematician, Crew Systems \& Aviation Operations Branch, Mail Stop 156A.

${ }^{6}$ Senior Analyst, Crew Systems \& Aviation Operations Branch, Mail Stop 156A.
}

1

American Institute of Aeronautics and Astronautics 
3000 feet with Precision Runway Monitoring (PRM) and offset Instrument Landing System (ILS) $)^{5}$. Dependent approaches to parallel runways are also currently approved for runways with centerline spacing of 2500 feet to 4300 feet, with the dependency of maintaining a minimum diagonal (aircraft-to-aircraft) separation distance of 1.5 nautical miles $(\mathrm{nm})$ or greater to mitigate the risk of wake encounters and aircraft collisions. Finally, current rules also allow for simultaneous, offset, instrument approaches (SOIA) to runways with centerline spacing between 750 and 3000 feet, but this procedure includes a visual approach segment for the trailing aircraft, and is not feasible under IMC. The objective of the SAPA concept is to explore the feasibility of allowing dependent parallel approaches to runways as close as 750 feet apart under IMC. The proposed SAPA procedure is designed for paired aircraft to remain nominally abreast of one another along the parallel approach, to ensure that each aircraft remains ahead of the other's trailing wake vortices ${ }^{4}$. Some flexibility exists since either aircraft can assume a leading or trailing position during the approach. Additionally, the SAPA procedure allows the initial (i.e. at the start of the SAPA procedure) trailing aircraft to over-take the initial lead aircraft prior to landing. This provides operational flexibility in the pairing of SAPA aircraft with differing approach speed profiles. To accomplish these capabilities, the SAPA concept requires autopilot coupling between paired aircraft, along with either: precision Ground-Based Augmentation System (GBAS) or Space-Based Augmentation System (SBAS) guidance.

The FAA and NASA Langley Research Center (LaRC) collaborated on this research study to assess the SAPA concept's preliminary operational feasibility, as it relates to wake vortex encounter avoidance. Results of this study were intended to support near-term investment decisions by the FAA. The results from this study are presented in this paper, including the initial characterization of a wake-free safe zone for the SAPA concept.

\section{Motivation and Experimental Setup}

A primary concern with closely-spaced parallel runway operations is the potential for wake encounters due to the close lateral spacing of aircraft. Therefore, it is important to fully understand the regions where the probability for wake encounters is greatly reduced. The research objective of this study is to determine, with a high degree of reliability, the location of a wake-free safe zone (WFSZ) for the SAPA concept, under various conditions including: various runway centerline spacings, staggered and un-staggered runway thresholds, and crosswinds.

Current-day instrument operations, with regard to potential wake encounters, are aimed at maintaining a relative aircraft separation so that the trailing aircraft remains safely behind and/or above the lead aircraft's wake ${ }^{5}$. In the SAPA concept, the relative spacing objective changes fundamentally such that the trailing aircraft is now attempting to remain a safe distance ahead of the lead aircraft's wake region (i.e. inside the WFSZ), along its parallel approach course, as shown in Figure 1. In the presence of variable crosswinds, a triangular wake region can be visualized as translating with a lead aircraft, which is generating a wake. A WFSZ can be defined as the distance, measured parallel to the runway centerline, from the lead aircraft to the trailing aircraft, where the probability of wake encounter by the trailing aircraft is sufficiently small as it remains ahead of the leader's wake. The SAPA Concept also provides the operational flexibility where the aircraft that is initially trailing at the start of the SAPA Procedure, may pass the other aircraft during the approach, and prior to landing. In this instance, the aircraft that is passed must now remain safely ahead of the wake vortices generated by the passing aircraft throughout the procedure. The goal of this study is to characterize the size of the WFSZ. In this study, it is assumed that the trailing aircraft is safely in the WFSZ at the start of the SAPA procedure.

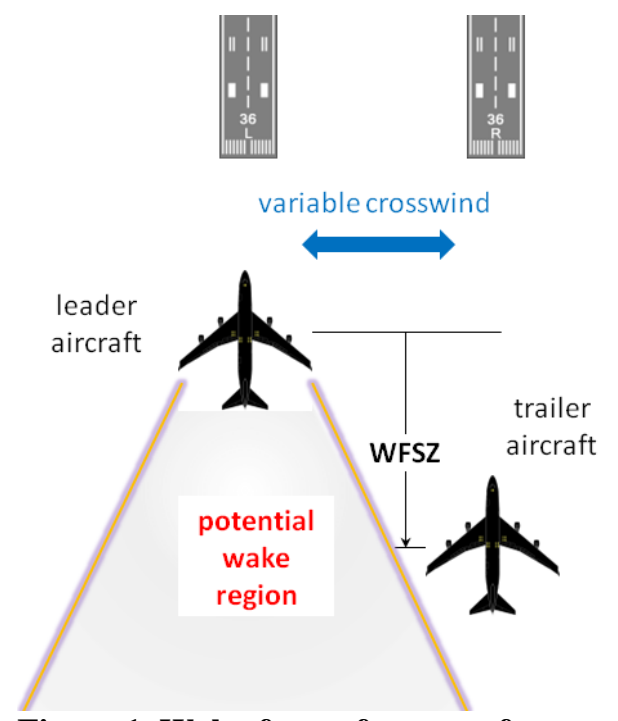

Figure 1. Wake-free safe zone reference.

\section{Experiment Design}

\section{A. Analysis Tools}

The modeling and simulation tool used in this study is the Wake Vortex Simulation and Analysis Tool

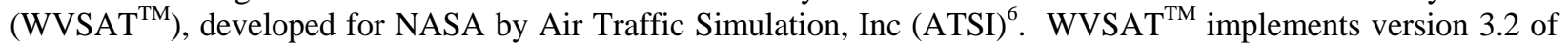
the Aircraft VOrtex Spacing System (AVOSS) Prediction Algorithm (APA), together with an aircraft simulation and 
visualization interface, that allows for fast prototyping of various flight profiles, with the capability for wake generation and detection. Some of the capabilities of $\mathrm{WVSAT}^{\mathrm{TM}}$ include: fast prototyping of experiments through a drag-and-drop modular design, Monte Carlo simulations, 3-dimensional visualization for interactive evaluation of scenarios, and the ability to evaluate wake behavior for a broad fleet of aircraft.

The APA version 3.2 model implemented within WVSAT $^{\mathrm{TM}}$ - originally developed by NASA LaRC - predicts the trajectory and circulation decay for a pair of vortices within a plane perpendicular to the generating aircraft's flight path ${ }^{7}$. WVSAT then interpolates between successive perpendicular planes, generated at the data sampling rate prescribed by the researcher (typically between one to five times per second), to produce a time history for each wake vortex. The APA algorithm can be characterized by three distinct stages: vortices that are generated out of ground-effect (OGE), near ground-effect (NGE), and in ground-effect (IGE). The OGE stage, models the wake movement and decay solely as a function of the atmospheric conditions, including wind, temperature, and turbulence levels. This typically results in a pair of vortices that descend while maintaining a nearly constant lateral separation relative to one another, as they translate (together) laterally at the approximate rate of any prevailing crosswind. In the NGE stage, the wake begins to interact with the ground, and modeling this interaction introduces simulated image vortices that analytically create a zero vertical velocity condition at the ground level, resulting in a lateral spreading between the two vortices. In the final stage, IGE, secondary vortices are induced by the primary vortices' interaction with the ground. This results in a further increase in the lateral separation rate between the primary wake vortices when compared with the NGE stage.

\section{B. Experimental Setup}

The experimental setup for this study consists of a paired-approach scenario to closely-spaced parallel runways typical of a SAPA approach. Two Heavy class aircraft, with characteristics similar to those of a Boeing 747-8F chosen for the pronounced wake characteristics compared to smaller aircraft - are used in an initial leader/follower configuration to a pair of closely-spaced parallel runways. The lead aircraft, on approach to the left runway, is considered the wake generator in WVSAT while the trailing aircraft, on approach to the right runway, is considered the wake detector, as depicted in Figure 2a). Each simulation case, consisting of a set of 2500 Monte Carlo runs for each scenario, begins with the trailing aircraft at an initial in-trail distance (ITD) relative to the lead aircraft. Each initial ITD is selected from a uniform distribution, spanning a pre-defined in-trail window (ITW.) The ITW is sized to explore the region of initial ITDs where wake encounters are detected for some of the 2500 Monte Carlo runs, but not for others. Analysis of the WVSAT wake encounter data then indicates the location or size of the WFSZ in terms of initial ITD.

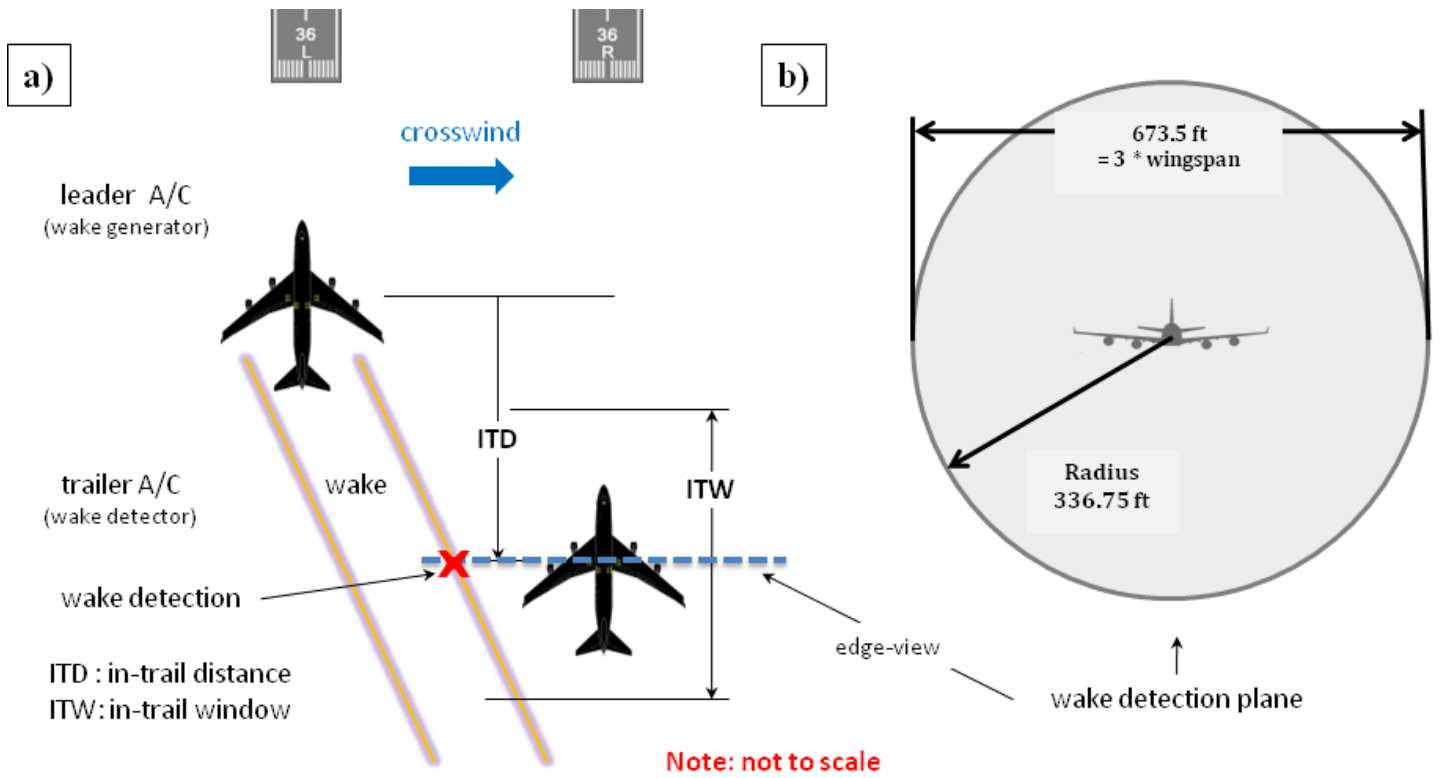

Figure 2. a) Aircraft approach simulation set-up. b) Reference wake detection plane.

Within WVSAT, the wake detector aircraft carries a wake detection surface (also called the area-of-interest (AOI)), perpendicular to its longitudinal axis and centered about its center of gravity (CG), as depicted in Figure 2a). Wake vortex elements, generated by the lead aircraft, which penetrate this AOI are recorded as potential wake encounters for the trailing aircraft. The AOI is sized conservatively to account for uncertainty related to the use of a 
fast-time, calibrated engineering model (i.e. APA) to represent complex wake dynamics. The user configurable AOI for this experiment is a circular area with a diameter equal to 3 wingspans, or 673.5 feet, and can be seen in Figure 2b). The crosswind used in this experiment is a constant 15 knots, measured perpendicular to the flight path and in the direction of the trailing aircraft's approach path. A 15 knot condition was selected as a reference crosswind condition such that the corresponding WFSZ would be valid for a large fraction of total aircraft approaches (i.e. those of 15 knots or less) in the NAS. Note, however, that tailwind effects are not considered in this study and the Aeronautical Information Manual (AIM) identifies light quartering tailwinds as requiring the maximum caution for trailing aircraft ${ }^{8}$.

Because of the scope of this experiment, the simulated SAPA approach used begins at the final approach fix (FAF) and ends at the runway, even though an operational version of the SAPA approach pairing may begin much earlier than this point. An attempt is made to create a realistic speed and altitude profile using experience and some consultation with two captains from a major US carrier (Figure 3). The altitude profile for this experiment is a constant three degree approach slope that nominally intercepts the runway threshold at the threshold crossing height (Table 1). The leader aircraft is simulated to have a light loading, with a weight of 425,000 pounds and a slower final approach speed than the trailing aircraft, a heavily-loaded aircraft with a weight of 749,000 pounds.

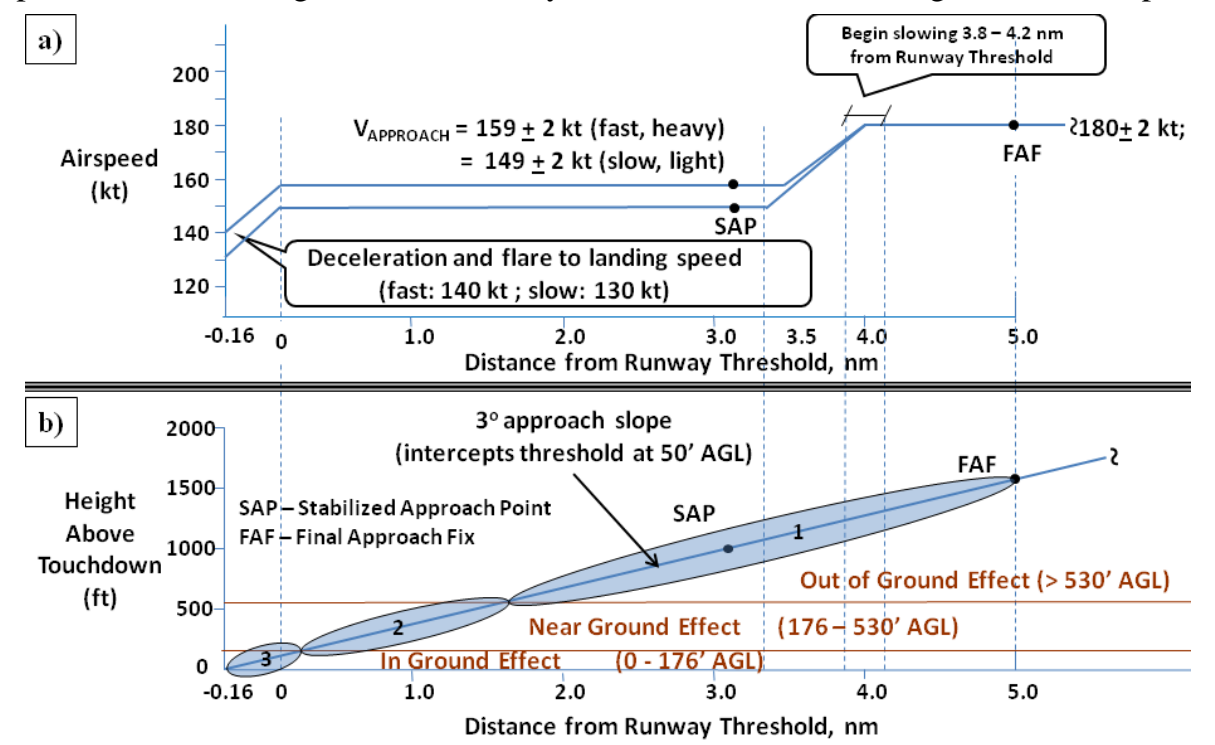

Figure 3. a) Speed profiles for leader and trailer aircraft. b) Altitude profile and simulation scenario regions.

Vertical and horizontal navigational errors are introduced in the form of offset values from the nominal approach trajectory. Uniform distributions of vertical error and cross-track error, consistent with RNP and WAAS LPV-200 standards ${ }^{[4]}$, are used to offset the constant 3 degree approach trajectory, while maintaining an approach parallel to the runway centerline. Each aircraft is allowed to converge to the runway centerline at approximately $0.3 \mathrm{~nm}$ from the runway threshold. Small speed variations are also used in the simulated approach profiles of both aircraft (Table 1).

The simulation scenarios are divided into three altitude regions as shown in Figure 3b). Each region corresponds to the three wake altitude regions modeled within the APA algorithm: out of ground-effect ( $>530$ feet), near ground-effect (176-530 feet), and in ground-effect. This segmentation of the overall approach profile allows for easier identification of the WFSZ in each of these regions, without the need for significant post-processing.

Experiments were conducted for three scenarios (altitude regions), three runway lateral separations (RLS), and two runway staggers (RS). The RLS used were 750, 1000 and 1400 feet and the RS were 0 and 1500 feet. Figure 4 shows how the RS and RLS are defined for this study. The overall experimental matrix resulted in 18 unique simulation cases, each of which consisted of 2500 Monte-Carlo simulation runs,

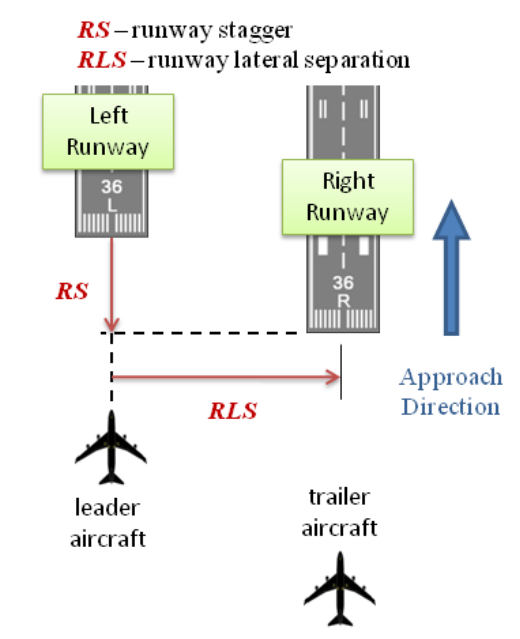

Figure 4. Runway stagger and runway lateral separation definitions.

4

American Institute of Aeronautics and Astronautics 
where one simulation run is defined as one pair of leader-trailer aircraft on a simulated approach.

Table 1. Experiment Parameters.

\begin{tabular}{|c|c|c|}
\hline Design Parameter & Units & Range \\
\hline Runway centerline spacing & $\overline{\mathrm{ft}}$ & $750,1000,1400$ \\
\hline Runway threshold stagger & $\mathrm{ft}$ & 0,1500 \\
\hline Crosswind speed & kt & 15 \\
\hline Initial longitudinal spacing between aircraft & $\mathrm{ft}$ & Variable \\
\hline Aircraft speed difference & kt & +10 (nominally) \\
\hline Aircraft pair types & N/A & Loaded -Heavy/Empty -Heavy \\
\hline Wake Detection Surface & Shape/ Size & Circle, Diameter $=3^{*}$ Span \\
\hline Simulation time step & Sec. & 1 \\
\hline \multicolumn{3}{|c|}{ Initial Conditions at the Final Approach Fix (FAF), and Stabilized Approach Point (SAP): } \\
\hline Cross-track error & $\mathrm{ft}$ & Uniform(-49.2, 49.2) \\
\hline Vertical error & $\mathrm{ft}$ & Uniform(-13.1, 13.1) \\
\hline Approach slope angle & Degrees & Constant $3.0^{\circ}$ \\
\hline \multirow{2}{*}{ Lead A/C IAS } & \multirow{2}{*}{$\mathrm{ft}$} & Uniform $(178,182) @$ FAF \\
\hline & & Uniform $(147,151) @$ SAP \\
\hline \multirow[b]{2}{*}{ Trailing A/C IAS } & \multirow[b]{2}{*}{ kt } & Uniform $(178,182) @$ FAF \\
\hline & & Uniform $(157,161) @$ SAP \\
\hline SAP Slow-Down Initiation Distance & $\mathrm{nm}$ & Uniform(3.8, 4.2) \\
\hline \multicolumn{3}{|l|}{ Landing Conditions: } \\
\hline Threshold Crossing Speed & $\mathrm{kt}$ & Equal to SAP speed \\
\hline \multirow{2}{*}{ Landing Speed } & \multirow{2}{*}{ kt } & Leader: Normal(130, 1.2) \\
\hline & & Trailer: Normal(140, 1.2) \\
\hline Threshold Crossing Ht. & $\mathrm{ft}$ & Constant $50+$ Navigational Vertical Error \\
\hline
\end{tabular}

\section{Scenario Details}

OGE Scenario: The OGE scenario was developed to explore the extent of the WFSZ outside of ground-effect. The primary characteristics of the wake model in this region are two main vortices with nearly constant lateral separation and decreasing wake descent rate as the vortices dissipate. Lateral transport of wakes in this region is dominated by the cross-component of the wind.

The simulation begins with the lead aircraft at the FAF - located at 5 nautical miles (nm) from the runway threshold - on approach to the left runway and with the trailing aircraft on approach to the right runway at an initial in-trail distance behind, both with a nominal indicated airspeed of 180 knots (+/- 2 knots error). The crosswind is a constant 15 knots blowing from left to right, perpendicular to the approach paths. Both aircraft maintain their initial airspeed until reaching a slow-down point located nominally at $4.0 \mathrm{~nm}(+/-0.2 \mathrm{~nm})$ from each aircraft's respective runway threshold. At the slow-down point, both aircraft begin slowing to a planned final approach speed (PFAS) before reaching the stabilized approach point (SAP), which is an airline-specific operational limitation defining the point on the approach where an aircraft must be fully configured to land, and selected to be at $3.3 \mathrm{~nm}$ from the runway threshold for this experiment. The remainder of the approach is conducted at the PFAS speed of each aircraft - 149 knots for the leader and 159 knots for the trailing aircraft (each with +/- 2 knots error) - until each aircraft reaches the OGE altitude boundary of 530 feet AGL, at which point the simulation of each aircraft is terminated. During each simulation run, the two aircraft are released with the trailing aircraft at a given in trail distance and the AOI is monitored to determine whether or not a wake encounter occurred.

The initial in-trail distance window for each runway lateral spacing was chosen to ensure that the boundary of the WFSZ would be explored, such that some runs would result in encounters and other runs would be clear of encounters. The initial in-trail distance between the leading and the trailing aircraft at the beginning of each run was varied uniformly within the following windows:

Table 2. OGE Scenario Initial In-Trail Windows.

\begin{tabular}{cc}
\hline Runway Lateral Spacing & Initial In-Trail Distance Window \\
\hline \hline $750 \mathrm{ft}$ & $0.3 \mathrm{~nm}-1.3 \mathrm{~nm}$ \\
$1000 \mathrm{ft}$ & $0.8 \mathrm{~nm}-1.8 \mathrm{~nm}$ \\
$1400 \mathrm{ft}$ & $1.6 \mathrm{~nm}-2.6 \mathrm{~nm}$ \\
\hline
\end{tabular}


NGE Scenario: The NGE scenario simulates only a small part of the overall approach trajectories of the leading/trailing aircraft. The NGE region is characterized by increased lateral separation of the two wake vortices and with further reduced vertical descent rate due to the proximity of the ground. Lateral wake movement is still primarily dominated by the cross-component of the wind.

The simulation begins with the leading aircraft at $530 \mathrm{ft}$ AGL at the slower PFAS of 149 knots (+/- 2 knots) and the trailing aircraft at a PFAS of 159 knots (+/- 2 knots) at some ITD behind. Both aircraft fly a constant speed to an altitude of $176 \mathrm{ft}$ AGL and the simulation run is terminated.

The initial in-trail distance for each simulation run is selected from the uniform distributions in Table 3 . Note that, as a result of a limitation of the WVSAT tool, the in-trail distance can only be defined relative to a fixed point for this experiment (the runway threshold is used). Since the leading aircraft is required to begin at a specified initial altitude of $530 \mathrm{ft}$, and a vertical navigational error is used in the simulated approach profile, the starting position of the leader is variable relative to the runway threshold. Thus, the result is that the "effective" in-trail window seen in the simulation is slightly larger than the prescribed in-trail window by approximately $500 \mathrm{ft}$; the differences between the prescribed and the effective in-trail windows can be seen in Table 3 .

Table 3. NGE Scenario Initial In-Trail Windows.

\begin{tabular}{ccc}
\hline Runway Lateral Spacing & Initial In-Trail Distance Window & Effective Initial In-Trail Distance Window \\
\hline \hline $750 \mathrm{ft}$ & $0.1 \mathrm{~nm}-1.1 \mathrm{~nm}$ & $0.0576 \mathrm{~nm}-1.1412 \mathrm{~nm}$ \\
$1000 \mathrm{ft}$ & $0.6 \mathrm{~nm}-1.6 \mathrm{~nm}$ & $0.5589 \mathrm{~nm}-1.6412 \mathrm{~nm}$ \\
$1400 \mathrm{ft}$ & $1.4 \mathrm{~nm}-2.4 \mathrm{~nm}$ & $1.3588 \mathrm{~nm}-2.4412 \mathrm{~nm}$ \\
\hline
\end{tabular}

IGE Scenario: The IGE scenario was designed to explore the effects on the WFSZ from wakes generated within the ground-effect region. These effects are typically characterized by higher lateral wake transport velocities that can have a significant impact on the size of the WFSZ.

For this scenario, the added flexibility provided by the SAPA concept is used to simulate the trailing aircraft passing the original leading aircraft. As a result, the new leading aircraft (and also the new wake generating aircraft) is now the more heavily loaded aircraft on approach to the right runway and the new trailing aircraft is now the lightly loaded aircraft on approach to the left runway. In addition, for the purposes of simulating a worst-case condition, the wind direction is also switched to be blowing from right to left in this scenario. The new leader begins the approach to the right runway at an altitude of $176 \mathrm{ft}$ AGL and at the PFAS of 159 knots (+/- 2 knots). The leader continues the approach to a touchdown at $1800 \mathrm{ft}$ from the runway threshold, slowing to the landing speed of approximately 140 knots at the threshold crossing height. The trailing aircraft begins at an initial in-trail distance behind the new leader at a PFAS of 149 knots (+/- 2 knots) and flies to a touchdown at $1800 \mathrm{ft}$ from the runway threshold at a landing airspeed of approximately 130 knots and the simulation run terminates.

The initial in-trail distance for each simulation run is selected from the uniform distributions in Table 4. As with the NGE scenario, the IGE scenario requires a specific aircraft initiation altitude (rather than a distance), which has the effect of increasing the size of in-trail windows used in the simulation, compared to the prescribed in-trail windows. The differences between the prescribed and effective in-trail windows can be seen in Table 4 . Note that Table 4 is presented in feet rather than nautical miles because a window size of $2000 \mathrm{ft}$ was used for this scenario, compared to the one nm window used in the OGE and NGE scenarios.

Table 4. IGE Scenario Initial In-Trail Windows.

\begin{tabular}{ccc}
\hline Runway Lateral Spacing & Initial In-Trail Distance Window & Effective Initial In-Trail Distance Window \\
\hline $750 \mathrm{ft}$ & $800 \mathrm{ft}-2,800 \mathrm{ft}$ & $550 \mathrm{ft}-3050 \mathrm{ft}$ \\
$1000 \mathrm{ft}$ & $1,500 \mathrm{ft}-3,500 \mathrm{ft}$ & $1250 \mathrm{ft}-3750 \mathrm{ft}$ \\
$1400 \mathrm{ft}$ & $3,500 \mathrm{ft}-5,500 \mathrm{ft}$ & $3250 \mathrm{ft}-5750 \mathrm{ft}$ \\
\hline
\end{tabular}

Additional operational conservatism was briefly explored in this study, by defining the WFSZ solely in terms of the lateral transport of the leader's wake into the path of the trailing aircraft and ignoring the vertical wake transport component. This is conservative because, in many cases, although the lead aircraft's wake translates laterally into the trailer's approach path, it descends below the circular AOI, resulting in no detected encounter. This conservative approach then, results in an altitude-independent characterization of the WFSZ that provides additional flexibility to the SAPA procedure by removing any vertical requirement for the pairing of the aircraft. To explore this, the circular AOI was replaced with a rectangular AOI having the same lateral dimension as the circular AOI, but that extended $1000 \mathrm{ft}$ above and below the trailing aircraft (1000 ft is the intended vertical separation for the SAPA concept, just prior to the trailing aircraft entering the WFSZ.) For conciseness, the results from these additional 
simulation runs are not further discussed in this paper but the effect was a reduction in the size of the WFSZ by four to five percent.

\section{Results}

\section{A. Limitations}

In the results and discussion below, the terminology "wake encounter" simply refers to the fact that the trailing aircraft's detection AOI detected a wake and that there was the "potential" for a wake encounter. This distinction of a "potential" for a wake encounter versus an "actual" wake encounter must be made, given that these are experimental results from a simulation. The detection AOI is a well-defined and conservatively-sized area and the wake position comes from a deterministic engineering model, both attempting to account for uncertainties in the true position of the wake. Given this distinction, the term "wake encounter" is used to simplify the discussion to follow.

The WFSZ characterizations presented here are from observed experimental results. Each of the 18 simulation scenario cases were conducted only once in a Monte-Carlo fashion and were not repeated. No attempt was made, at this time, to calculate the statistical probability of encounter based upon the simulation results. Rather, the observed minimum in-trail distance with a wake encounter was selected to define the approximate size of the WFSZ.

The results presented here are highly dependent on the speed profile and aircraft configurations simulated. Characterization of the WFSZ is based solely on a binary wake/no-wake encounter for a given initial in-trail distance. Other speed profiles would provide a different wake encounter distribution for the same initial in-trail window. This is due to the differing levels of in-trail distance compression and/or expansion between the aircrafts' relative positions. The wake modeling is primarily dependent on aircraft weight, wingspan and airspeed; consequently, varying these parameters would result in different WFSZ characteristics.

\section{B. Scenario 1 - OGE}

A sample of the results for the OGE scenario can be seen in Figure 5 and Figure 6. Figure 5 shows simulation results for the $750 \mathrm{ft}$ runway lateral separation with no runway stagger case and Figure 6 shows the results for the $750 \mathrm{ft}$ runway lateral separation with a $1500 \mathrm{ft}$ runway stagger. Figures for the other OGE cases are not presented here, for conciseness, but a summary of the results can be found in the Discussion section.

For each simulation case, two figures are shown. The top figure (a) shows the binned (by range of in-trail distances) number of simulation runs from the pool of 2500 Monte-Carlo runs that had a wake encounter. The bottom figure (b) shows the percentage of runs that had encounters based on the number of runs released within a given initial in-trail distance bin. The $\mathrm{x}$-axis limits for each figure are the in-trail window limits used in the simulation for the respective scenario.
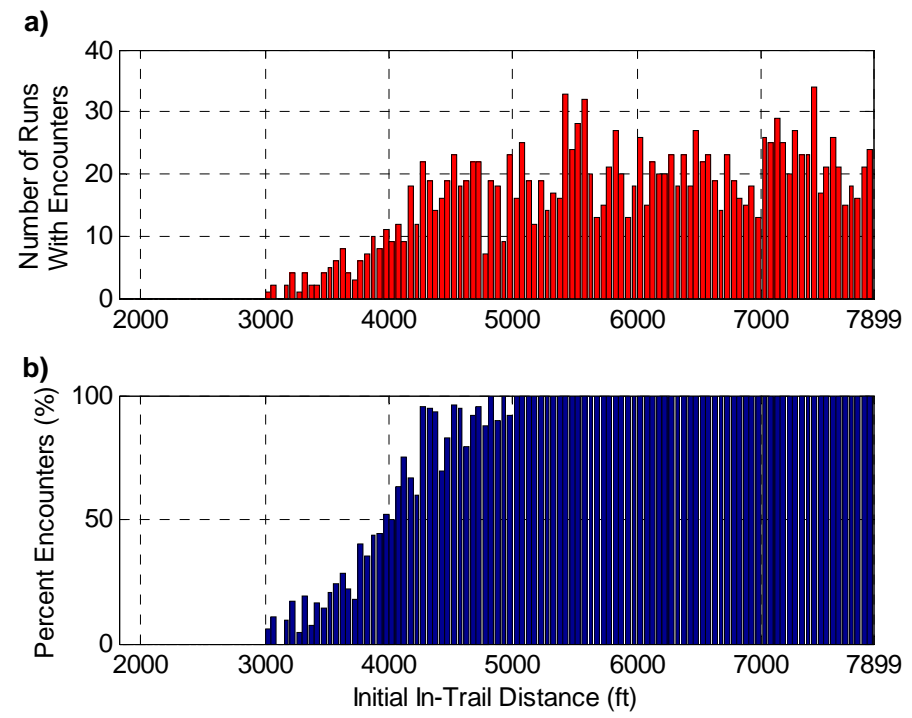

Figure 5. Scenario 1 results, OGE, $750 \mathrm{ft}$ RLS, no RS, $50 \mathrm{ft}$ bins; a) number of simulation runs with encounters, $b$ ) percentage of runs with encounters. 

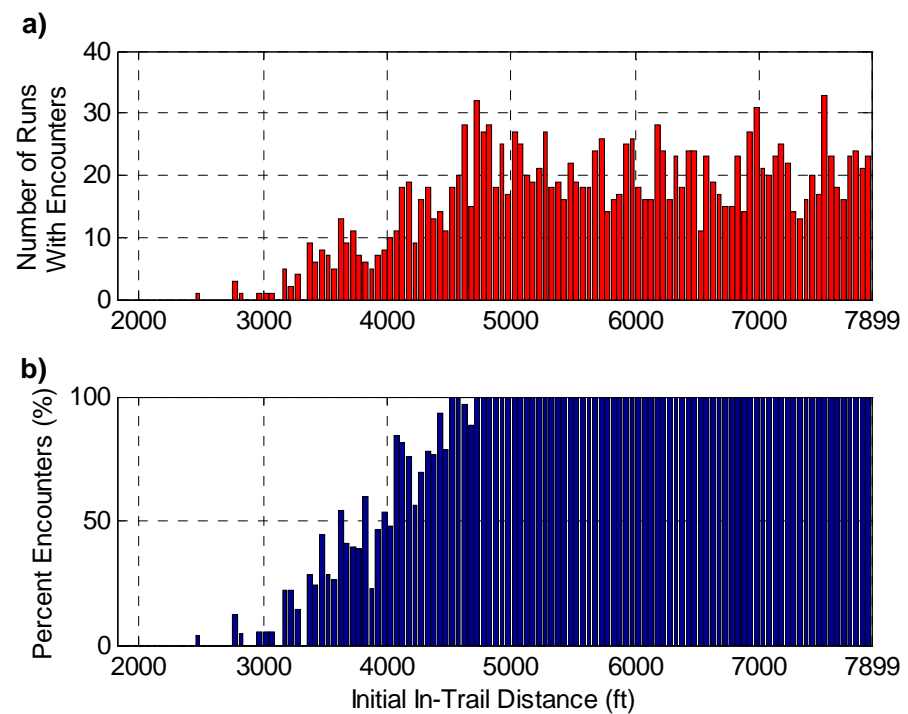

Figure 6. Scenario 1 results, OGE, $750 \mathrm{ft}$ RLS, $1500 \mathrm{ft}$ RS, $50 \mathrm{ft}$ bins; a) number of simulation runs with encounters, $b$ ) percentage of runs with encounters.

\section{Scenario 2 - NGE}

Figure 7 and Figure 8 are plots for the results of the NGE scenario for a $750 \mathrm{ft}$ runway lateral separation, with no runway stagger and with a $1500 \mathrm{ft}$ runway stagger, respectively. The results for other NGE scenarios are summarized in the Discussion section.

As with the OGE scenario, two figures are shown for each simulation case. The top figure (a) shows the binned number of simulation runs from the pool of 2500 Monte-Carlo runs that had a wake encounter. The bottom figure (b) shows the percentage of runs that had encounters based on the number of runs released within a given initial intrail distance bin. The $\mathrm{x}$-axis limits for each figure are the in-trail window limits used in the simulation for the respective scenario.
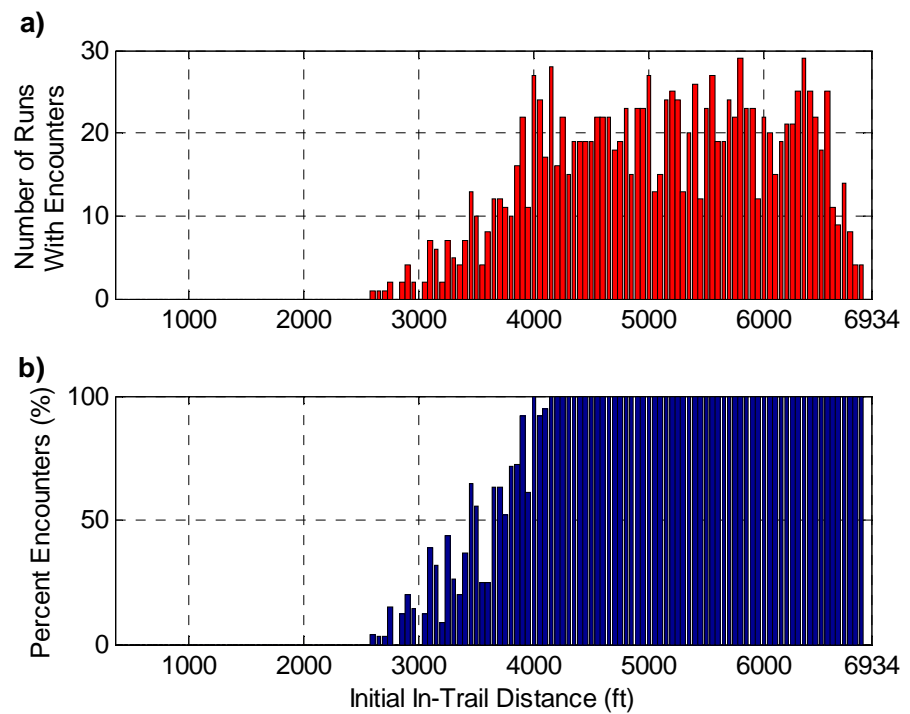

Figure 7. Scenario 2 results, NGE, $750 \mathrm{ft}$ RLS, no RS, $50 \mathrm{ft}$ bins; a) number of simulation runs with encounters, b) percentage of runs with encounters. 

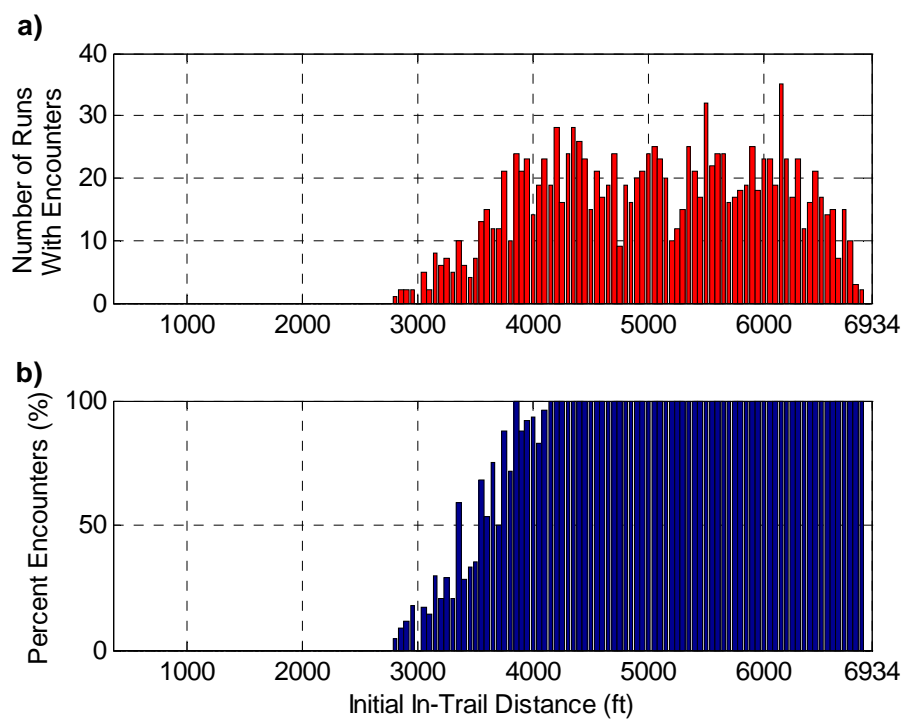

Figure 8. Scenario 2 results, NGE, $750 \mathrm{ft}$ RLS, $1500 \mathrm{ft} \mathrm{RS,} 50 \mathrm{ft}$ bins; a) number of simulation runs with encounters, $b$ ) percentage of runs with encounters.

\section{Scenario 3 - IGE}

Figure 9 and Figure 10 are plots for the results of the IGE scenario for a $750 \mathrm{ft}$ runway lateral separation, with no runway stagger and with a $1500 \mathrm{ft}$ runway stagger, respectively. The results for other IGE scenarios are summarized in the Discussion section.

As with the OGE and NGE scenarios, two figures are shown for each simulation case. The top figure (a) shows the binned number of simulation runs from the pool of 2500 Monte-Carlo runs that had a wake encounter. The bottom figure (b) shows the percentage of runs that had encounters based on the number of runs released within a given initial in-trail distance bin. The x-axis limits for each figure are the in-trail window limits used in the simulation for the respective scenario.
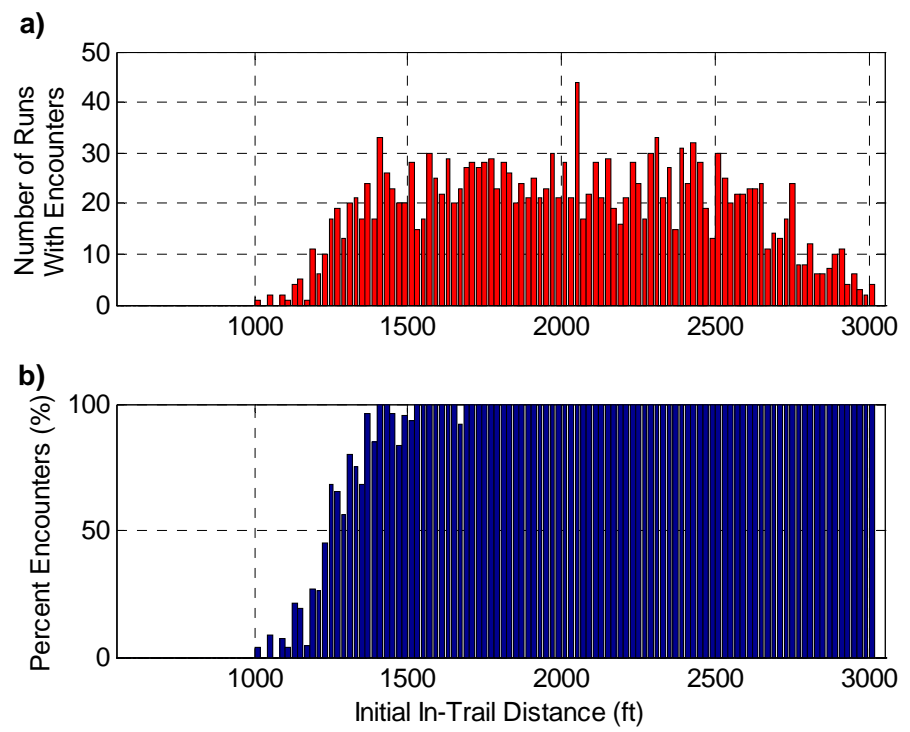

Figure 9. Scenario 3 results, IGE, $750 \mathrm{ft}$ RLS, no RS, $20 \mathrm{ft}$ bins; a) number of simulation runs with encounters, $b$ ) percentage of runs with encounters. 

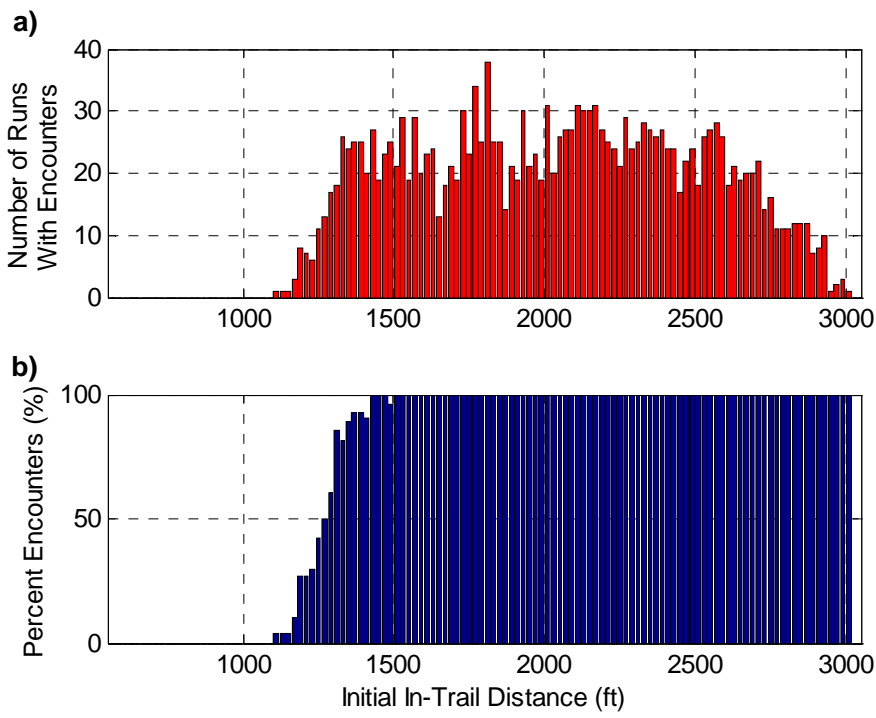

Figure 10. Scenario 3 results, IGE, $750 \mathrm{ft}$ RLS, $1500 \mathrm{ft} \mathrm{RS,} 20 \mathrm{ft}$ bins; a) number of simulation runs with encounters, $b$ ) percentage of runs with encounters.

\section{E. Discussion}

The results show similar characteristics between the three simulation scenarios but different boundaries for the WFSZ. In every scenario case (of the 18 cases simulated), there is an initial in-trail distance beyond which 100 percent of the runs have observed wake encounters. Likewise, there is an initial in-trail distance ahead of which none of the runs have observed wake encounters. Between these two distances lies the WFSZ "frontier," which is of particular interest for this experiment because it helps to define the probability for wake encounters at every initial in-trail distance. Subplot b) in Figure 5-Figure 10 presents the observed percentage of runs with wake encounters. Subplot b), therefore, represents an empirical estimate of the cumulative density function (CDF) for the probability of wake encounters as a function of initial in-trail distance. Figure 6b) suggests that a significantly higher number of samples from the left tail of the WFSZ frontier region would be required to more accurately calculate the statistical CDF from the experimental data, due to lack of wake encounters in some distance bins. Thus, the first-order estimate of the WFSZ location, for this experiment, is determined simply by observing the smallest in-trail distance having an observed wake encounter from each Monte Carlo set of runs. The first-order WFSZ estimates for each of the 18 scenario cases are listed in Table 5 and shown graphically in Figure 11.

Table 5. Smallest Initial In-Trail Distances for Each Scenario and Case with Observed Wake Encounters (ft).

\begin{tabular}{l|ccc|ccc}
\hline \multirow{2}{*}{\begin{tabular}{l} 
Runway Lateral \\
\cline { 2 - 7 } Separations
\end{tabular}} & \multicolumn{3}{|c|}{ No Runway Stagger } & \multicolumn{3}{c}{ Runway Stagger (1500 ft) } \\
\cline { 2 - 7 } OGE & $750 \mathrm{ft}$ & $1000 \mathrm{ft}$ & $1400 \mathrm{ft}$ & $750 \mathrm{ft}$ & $1000 \mathrm{ft}$ & $1400 \mathrm{ft}$ \\
NGE & 3044 & 6026 & 11172 & 2477 & 5824 & 10717 \\
IGE & 2621 & 5374 & 9802 & 2799 & 5385 & 9383 \\
\hline
\end{tabular}

Figure 11 illustrates the different sized WFSZs for each of the simulation scenario regions (OGE, NGE, and IGE). These preliminary characterizations of the WFSZ suggest that the safe zone is different for each of the three regions. The WFSZ is only slightly reduced in the NGE stage of the approach (compared to OGE) but is significantly reduced in the IGE stage. For example, for a Closely-Spaced Parallel Approach (CSPA) to a pair of runways with $750 \mathrm{ft}$ runway lateral separation and no runway stagger, the WFSZ changes from $3044 \mathrm{ft}$ OGE to 2621 $\mathrm{ft}$ NGE, and finally, to $1008 \mathrm{ft}$ IGE. Given that an aircraft will spend a relatively short amount of time in the NGE region, it would be very difficult, if not impossible, for most aircraft to close a gap of $\sim 1600 \mathrm{ft}$ in this amount of time. In fact, a calculation using the nominal speed profiles for this experiment showed that only $\sim 450 \mathrm{ft}$ of in-trail distance could be reduced using the speed differential of 10 knots between the start of the NGE and the start of the IGE regions. For a given runway spacing and stagger, from Figure 11 above, the set of three bars indicates the 


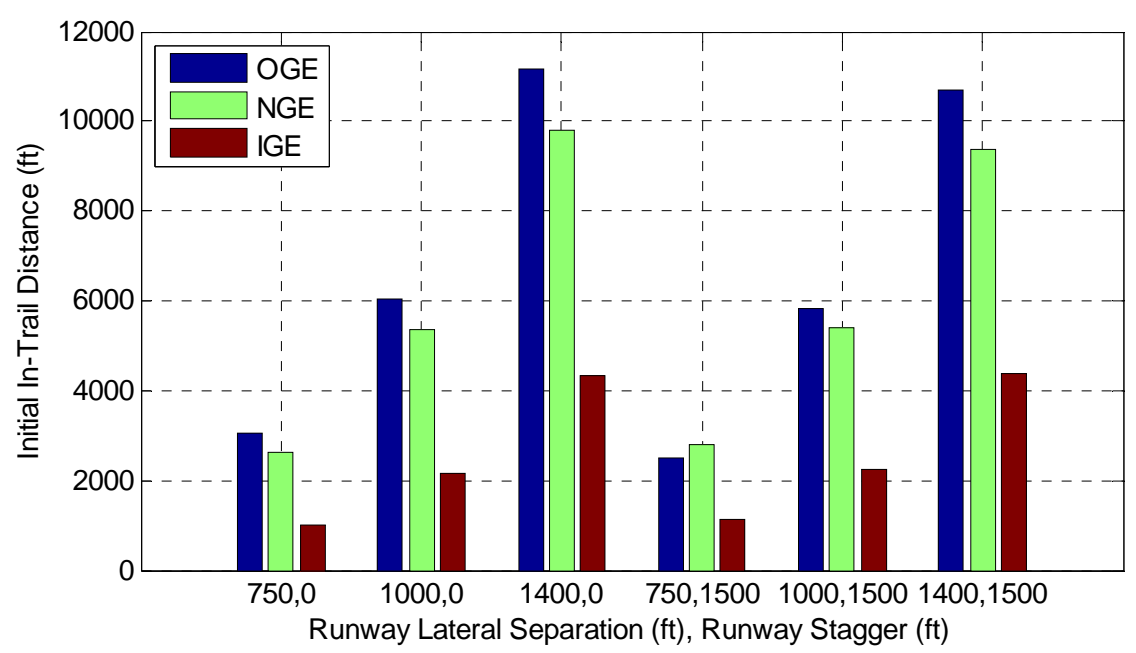

Figure 11. Smallest initial in-trail distances for each scenario and case with observed wake encounters. amount of in-trail distance that must be closed as the trailing aircraft transitions from OGE to NGE and finally to IGE. As previously noted, the large intrail gap closure required between NGE and IGE suggests that the trailing aircraft would need to close a substantial part of that gap prior to the transition between NGE and IGE.

For the conditions evaluated, runway stagger appears to only modestly reduce the WFSZ size in some cases, predominantly in the OGE scenario. The runway stagger approaches were conducted with the leading aircraft on high approach side (a worst-case condition). The effect of this higher approach profile for the leading aircraft is that the wake penetrates the wake detection surface at a relatively higher location along the perimeter of the AOI. Consequently, the effective separation between the leading aircraft and detecting aircraft's AOI is slightly smaller than with the no runway stagger cases, as shown in Figure 11.

The results shown in Figure 5-Figure 10 suggest the need for increased sampling (i.e., importance sampling) in the left tail of the WFSZ frontier region in order to more accurately calculate the wake encounter probability CDF. With this more accurate CDF, the WFSZ could then be defined in terms of a statistical probability of wake encounter of some very low value (e.g. $10^{-6}-10^{-9}$ ).

A few additional experimental runs were conducted to investigate the effects of Monte-Carlo sampling and the in-trail window size on the observed probability of wake encounters for one of the simulation scenarios. Figure 12 shows the results for scenario 1, OGE with $750 \mathrm{ft}$ runway lateral separation and no runway stagger. The figure includes the data from the original experiment for this same scenario case (green). A theoretical "worst-case" and a theoretical "most-optimistic" case were calculated by selecting the "worst-case" and "most-optimistic" values for the Monte Carlo parameters and varying only the initial in-trail distance. This allowed determination of the theoretical (deterministic) zero percent and 100 percent encounter probability at $1947 \mathrm{ft}$ and $5272 \mathrm{ft}$, respectively (based on the experimental distributions used in this study). Two additional experimental runs were conducted by selecting both a smaller in-trail window (higher run density, 2500 runs in [0.45 0.6$] \mathrm{nm}$ window) and a large number of runs in a low probability region (10000 runs in [0.325 0.475] nm window). The higher density runs showed CDF trends similar to those observed in the baseline experimental runs, as shown in Figure 12.

For this experiment, confidence intervals for the value of the observed WFSZ were calculated using an extreme value distribution methodology and are summarized in Table 6. These confidence intervals represent the intervals within which the observed WFSZ values should lie 99\% of the time if these same Monte Carlo experiments were repeated. It is important to note that these confidence intervals do not represent a $99 \%$ confidence of no wake encounters; instead, they serve to show that there is $99 \%$ confidence that the observed WFSZ values are within $151 \mathrm{ft}$ (worst-case) of the

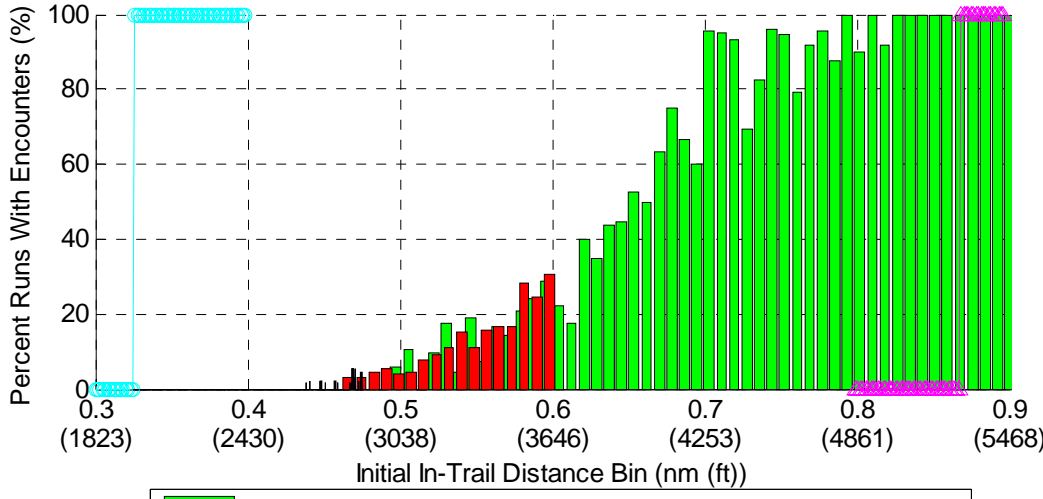

2500 Runs - [0.3 1.3] nm window 2500 Runs [0.45 0.6] nm window 2500 Runs - [0.3 0.4] nm window, Worst Case, MC IIT Only! 2500 Runs - [0.8 0.9] nm window, Most Optimistic, MC IIT Only! 10000 Runs - [0.325-0.475] nm window

Figure 12. Scenario 1, OGE, $750 \mathrm{ft}$ RLS, no RS, higher density data runs results. 
expected values, or less than one wingspan for this experiment. A more extensive discussion of the methodology used to calculate the confidence intervals can be found the SAPA study final report ${ }^{9}$.

Table 6. 99\% Asymptotic Confidence Intervals for Initial In-Trail Distance (Lower/Upper Limits, ft).

\begin{tabular}{c|ccc|ccc}
\hline \multirow{2}{*}{$\begin{array}{c}\text { Runway Lateral } \\
\text { Separations }\end{array}$} & \multicolumn{3}{|c|}{ No Runway Stagger } & \multicolumn{3}{c}{ Runway Stagger (1500 ft) } \\
\cline { 2 - 7 } & $750 \mathrm{ft}$ & $1000 \mathrm{ft}$ & $1400 \mathrm{ft}$ & $750 \mathrm{ft}$ & $1000 \mathrm{ft}$ & $1400 \mathrm{ft}$ \\
\hline \hline OGE & $2968 / 3044$ & $5979 / 6026$ & $11083 / 11171$ & $2409 / 2477$ & $5714 / 5824$ & $10653 / 10717$ \\
NGE & $2470 / 2621$ & $5265 / 5374$ & $9737 / 9802$ & $2707 / 2799$ & $5284 / 5385$ & $9306 / 9383$ \\
IGE & $990 / 1008$ & $2099 / 2141$ & $4252 / 4314$ & $1102 / 1115$ & $2216 / 2257$ & $4313 / 4360$ \\
\hline
\end{tabular}

\section{Conclusion}

A preliminary characterization of a wake-free safe zone (WFSZ) has been performed for the Simplified Aircraftbased Paired Approach (SAPA) concept. The experiment employed Monte-Carlo simulation of varying approach profiles by a pair of aircraft to closely-spaced parallel runways. Three different runway lateral spacings were investigated, along with no stagger and $1500 \mathrm{ft}$ runway stagger between runway thresholds. The paired aircraft were flown in a leader/trailer configuration with potential wake encounters detected using a wake detection surface translating with the trailing aircraft. The results suggest that the WFSZ can be characterized in two primary regions, in ground-effect and out of ground-effect, with the in ground-effect region being the limiting case with a significantly smaller WFSZ.

Additional experiments are required to more fully characterize the WFSZ. The preliminary characterization performed here suggests that importance sampling, concentrated in the very low-probability-of-wake-encounter region, would be required to generate a sufficiently accurate CDF to characterize the WFSZ. This would allow the definition of a WFSZ with a probability of wake encounter corresponding to the value, determined by the Federal Aviation Administration (FAA), that would be acceptable (e.g. $10^{-6}-10^{-9}$ ) for a SAPA-like procedure to be deployed. Given that the results presented here are specific to the conditions of this experiment, a wider range of speed profiles and aircraft configurations need to be studied to determine their effects on the WFSZ. Alternatively, a time-based WFSZ characterization (rather than an initial in-trail distance) may provide greater flexibility to procedure developers by specifying the WFSZ along the entire approach, rather than just at the FAF and runway threshold, as was done in this study.

\section{Acknowledgments}

The authors wish to thank NASA Langley Research Center (Crew Systems \& Aviation Operations Branch and Aeronautics Systems Analysis Branch), the Airspace Systems Program, and the Federal Aviation Administration for supporting this work under Interagency Agreement IA1-973, Technical Direction 2. Thanks to Fred Proctor, of NASA Langley Research Center, for taking the time to answer some of the authors' questions related to wake modeling and for consultation on this SAPA study. Thanks also to Fernando Rico-Cusi who helped to develop the baseline simulation scenarios and provided some of his expertise in the use of the WVSAT simulation tool.

\section{References}

\footnotetext{
${ }^{1}$ Viken, J., et. al., "NAS Demand Predictions, Transportation Systems Analysis Model (TSAM) Compared with Other Forecasts," $6^{\text {th }}$ AIAA Aviation Technology, Integration, and Operations Conference, AIAA-2006-7761, Wichita, Kansas, 25-27 September, 2006.

${ }^{2}$ Miller, M.E., Trott, G.A., "Effects of Future Traffic on the National Airspace System,” AIAA Modeling and Simulation Technologies Conference and Exhibit, AIAA-2004-5438, Providence, Rhode Island, 16-19 August, 2004.

${ }^{3}$ Decleene, Bruce and Swider, Christopher, "Simplified Aircraft-Based Paired Approach (SAPA)," Internal FAA concept paper, July 16, 2008.

${ }^{4}$ Closely Spaced Parallel Operation Working Group, Closely Spaced Parallel Operations Concept of Operations, draft, FAA, September 2009.

${ }^{5}$ Federal Aviation Administration, Aeronautical Information Manual (AIM), Effective 2/11/10, Chapter 5, Section 4, Arrival Procedures, http://www.faa.gov/air_traffic/publications/ATPubs/AIM/aim.pdf, accessed June 2010.

${ }^{6}$ www.atsi.aero

${ }^{7}$ Robins, R.R., Delisi, D.P., “NWRA AVOSS Wake Vortex Prediction Algorithm Version 3.1.1,” NASA/CR-2002-211746, June 2002.
} 
${ }^{8}$ Federal Aviation Administration, Aeronautical Information Manual (AIM), Effective 2/11/10, Chapter 7, Section 3, Safety of Flight, http://www.faa.gov/air_traffic/publications/ATPubs/AIM/aim.pdf, accessed June 2010.

${ }^{9}$ Johnson, Sally C., et. al., "Simplified Aircraft-based Paired Approach: Concept Definition and Initial Analysis," NASA Internal Report to the FAA, March 2010. 